doi: $10.15407 /$ ujpe62.02.0172

A.K. PRYKARPATSKY ${ }^{1,2}$

${ }^{1}$ Faculty of Applied Mathematics at AGH University of Science and Technology (Kraków, Poland)

2 Ivan Franko State Pedagogical University of Drohobych

(Drohobych, Lviv region, Ukraine)

\title{
THE QUANTUM FERMIONIC \\ CHARGED PARTICLE SELF-INTERACTION PROBLEM WITHIN THE FOCK MULTITIME AND FEYNMAN PROPER TIME PARADIGMS
}

$11.10 .-\mathrm{z} ; 11.15 .-\mathrm{q}$

11.10.Wx, 05.30.-d

\begin{abstract}
A quantum fermionic massless charged particle self-interacting with its own self-generated bosonic electromagnetic field is reanalyzed in the framework of the Fock multitime and Feynman proper time paradigms. The self-interaction phenomenon structure is studied within the method based on a suitably renormalized quantum Fock space. The fermionic charged particle mass spectrum is also discussed.

Ke ywords: quantum fermionic field, charged particle self-interaction problem, quantum Maxwell electrodynamics, Fock space, Fock multitime approach, charged particle inertial mass problem, least action principle, Lagrangian formalism, Feynman proper time paradigm.
\end{abstract}

\section{Introduction}

The elementary point charged particle (e.g., electron) mass problem inspired many physicists [43] from the past such as J.J. Thompson, G.G. Stokes, H.A. Lorentz, E. Mach, M. Abraham, P.A.M. Dirac, G.A. Schott, J. Schwinger, and others. Nonetheless, their studies have given no clear explanation of this phenomenon, which stimulated new researchers to tackle it from different approaches based on new ideas stemming both from the classical Maxwell-Lorentz electromagnetic theory, as in $[19,28,29,32,33,37,38$, $62,66,70]$, and modern quantum field theories of the Yang-Mills and Higgs types, as in [3,39,40,78], whose recent and extensive review appeared in [77].

In the present work, we mostly concentrate on the detailed quantum and classical analyses of the self-interacting shell model charged particle within the Fock multitime approach $[21,31]$ and the Feynman proper

(C) A.K. PRYKARPATSKY, 2017

172 time paradigm $[22,23,28,29]$ subject to deriving the electromagnetic Maxwell equations and the related expression for a Lorentz-like force within the vacuum field theory approach devised in works $[8,10,15-$ 18, 32-34]. Furthermore, we will explain and apply the obtained results to treating the classical LorentzAbraham $[1,44,45,48-52,54,57,58,60,65,67,73,74,76$, 79] electromagnetic mass origin problem. For the first time, the proper time approach to classical electrodynamics and quantum mechanics was apparently suggested in 1937 by V. Fock in work [30], where, in particular, an alternative proper-time-based Lagrangian description of a point charged particle in an external electromagnetic field was constructed. A more detailed motivation for using the proper-time approach was later presented by R. Feynman in his lectures [28]. Concerning the alternative and much later investigations of the a priori given quantum electromagnetic Maxwell equations in the Fock space, we mention the Gupta-Bleuer approach $[11,14,36]$ and other

ISSN 2071-0194. Ukr. J. Phys. 2017. Vol. 62, No. 2 
ones developed in $[7,25,70]$. As is well-known [7, 14], the first approach contradicts one of the most important field theoretical principles, namely, the positive definiteness of the quantum event probability, and is strongly based on making the nonphysical use of an indefinite metric on quantum states. The latter are completely non-relativistic and based on the canonical quantization scheme $[7,71]$ in the case of the Coulomb gauge condition. Inspired by these and related classical results, we have shown that the quantum mechanism of self-interaction of a charged particle with its self-generated electromagnetic field consists of two physically different phenomena, whose influence on the structure of the resulting Hamilton interaction operator appears to be crucial and gives rise [64] to a modified analysis of the related classical shell model of a charged particle within the Lagrangian formalism. As a result of our scrutiny of studying the classical electromagnetic mass problem, there was demonstrated that it can be satisfactorily solved within the classical H. Lorentz and M. Abraham arguments augmented with the additional electron stability condition, which was not taken before into account yet and appeared to be very important for balancing the related electromagnetic field and mechanical electron momenta. Their importance, following the recent works $[54,65,67]$ devoted to analyzing the electron charged shell model, was realized within the suggested pressure-energy compensation principle suitably applied to the ambient electromagnetic energy fluctuations and the self-generated electrostatic Coulomb electron energy. In the case of a point charged particle, the alternative relativistic invariant approach to studying the radiation reaction force was suggested earlier by C. Teitelboim [74]. This approach was based on a formal relativistic invariant splitting of the electromagnetic energy-momentum tensor and on the derivation of the related suitably renormalized equations of motion of a charged particle.

\section{Quantum Origin of the Charged Particle Self-Interaction}

Consider a free relativistic quantum fermionic a priori massless particle field described $[13,14]$ by means of the second-quantized self-adjoint Dirac-Weyl-type Hamiltonian

$H_{f}=\int_{\mathbb{R}^{3}} d^{3} x \psi^{+}\left\langle c \alpha, \frac{\hbar}{i} \nabla\right\rangle \psi$, where $\alpha \in \mathbb{E}^{3} \otimes$ End $M^{4}$ denotes the standard Dirac spin matrix vector representation in the Minkowski space $M^{4}, c \in \mathbb{R}_{+}$is the light velocity, $\langle\cdot, \cdot\rangle$ denotes the usual scalar product in the Euclidean space $\mathbb{E}^{3}$, $\psi: \mathbb{R}^{3} \rightarrow(\text { End } \Phi)^{4}$, which is a spinor of the quantum annihilation operators acting in a suitable Fock space $\Phi$ endowed with the usual scalar product $(\cdot, \cdot)$, and $\psi^{+}: \mathbb{R}^{3} \rightarrow(\text { End } \Phi)^{4}$ is the adjoint co-spinor of creation operators in the Fock space $\Phi$. The following anticommuting $[13,14]$ operator relations

$$
\begin{aligned}
& \psi_{j}(x) \psi_{l}^{+}(y)+\psi_{l}^{+}(y) \psi_{j}(x)=\delta_{j l} \delta(x-y), \\
& \psi_{j}(x) \psi_{l}(y)+\psi_{l}(y) \psi_{j}(x)=0, \\
& \psi_{j}^{+}(x) \psi_{l}^{+}(y)+\psi_{l}^{+}(y) \psi_{j}^{+}(x)=0
\end{aligned}
$$

hold for any $x, y \in \mathbb{R}^{3}$ and $j, l \in \overline{1,4}$, which are compatible with the related Heisenberg operator dynamics generated by the fermionic Hamiltonian operator (1):

$\partial \psi / \partial \bar{t}:=\frac{i}{\hbar}\left[H_{f}, \psi\right], \partial \psi^{+} / \partial \bar{t}:=\frac{i}{\hbar}\left[H_{f}, \psi^{+}\right]$

with respect to its own laboratory reference frame $\mathcal{K}_{\bar{t}}$ parametrized by the evolution parameter $\bar{t} \in \mathbb{R}$.

It is clear that Hamiltonian (1) possesses no information on the important characteristics of the electric charge $\xi \in \mathbb{R}$, which generates the own electromagnetic field interacting both with it and with other ambient charged particles. As is usually accepted, we will model a free electromagnetic field by its bosonic self-adjoint operator four-potential $(\varphi, A): \mathbb{R}^{3} \rightarrow$ $\rightarrow \operatorname{Hom}\left(\Phi, \Phi^{4}\right)$, whose evolution is generated by the self-adjoint Hamiltonian

$H_{b}=2 \int_{\mathbb{R}^{3}} d^{3} k|k|^{2}\left[\left\langle A^{+}(k), A(k)\right\rangle-\varphi(k) \varphi^{+}(k)\right]$

acting in the common Fock space $\Phi$ and represented by means of the field operators expanded by the Fourier transformation

$$
\begin{aligned}
& \varphi(x):=\frac{1}{(2 \pi)^{3 / 2}} \int_{\mathbb{R}^{3}} d^{3} k \varphi(k) \exp (i\langle k, x\rangle)+ \\
& +\frac{1}{(2 \pi)^{3 / 2}} \int_{\mathbb{R}^{3}} d^{3} k \varphi^{+}(k) \exp (-i\langle k, x\rangle), \\
& A(x):=\frac{1}{(2 \pi)^{3 / 2}} \int_{\mathbb{R}^{3}} d^{3} k A(k) \exp (i\langle k, x\rangle)+ \\
& +\frac{1}{(2 \pi)^{3 / 2}} \int_{\mathbb{R}^{3}} d^{3} k A^{+}(k) \exp (-i\langle k, x\rangle) .
\end{aligned}
$$


The coefficients of expansions (5) satisfy the following $[14,21,31]$ commutation operator relations:

$$
\begin{aligned}
& {\left[\varphi(k), \varphi^{+}(s)\right]=-\frac{c \hbar}{2|k|} \delta(k-s),} \\
& {\left[\varphi(k), A_{j}(s)\right]=0,} \\
& {[\varphi(k), \varphi(s)]=0=\left[\varphi^{+}(k), \varphi^{+}(s)\right],} \\
& {\left[A_{j}(k), A_{l}^{+}(s)\right]=\frac{c \hbar}{2|k|} \delta_{j l} \delta(k-s),} \\
& {\left[A_{j}(k), A_{l}(s)\right]=0=\left[A_{j}^{+}(k), A_{l}^{+}(s)\right]}
\end{aligned}
$$

for all $k, s \in \mathbb{E}^{3}$ and $j, l \in \overline{1,3}$, compatible with the related Heisenberg operator dynamics [14] generated by the electromagnetic field Hamiltonian (4):

$\frac{\partial A}{\partial \tilde{t}}:=\frac{i}{\hbar}\left[H_{b}, A\right], \frac{\partial \varphi}{\partial \tilde{t}}:=\frac{i}{\hbar}\left[H_{b}, \varphi\right]$

with respect to its own laboratory reference frame $\mathcal{K}_{\tilde{t}}$ parametrized by the temporal parameter $\tilde{t} \in \mathbb{R}$. In particular, based on the commutation relations (6), one can verify that the electric field

$E:=-\nabla \varphi-\frac{1}{c} \frac{\partial A}{\partial \tilde{t}}$

and the magnetic field

$B:=\nabla \times A$

satisfy the operator Maxwell equations in vacuum, and the following weak Lorentz-type constraints

$$
\begin{aligned}
& C_{0}(k) \Phi:=i[\langle k, A(k)\rangle-|k| \varphi(k)] \Phi=0, \\
& C_{0}^{+}(k) \Phi:=-i\left[\left\langle k, A^{+}(k)\right\rangle-|k| \varphi^{+}(k)\right] \Phi=0
\end{aligned}
$$

hold in the Fock space $\Phi$ for all $k \in \mathbb{E}^{3}$. Since the operators $C_{0}(k): \Phi \rightarrow \Phi$ and $C_{0}^{+}(k): \Phi \rightarrow \Phi$ and the Hamiltonian (4) are mutually commuting for all $k \in \mathbb{E}^{3}$, i.e.,

$$
\begin{aligned}
& {\left[C_{0}(k), C_{0}(l)\right]=0=\left[C_{0}(k), C_{0}^{+}(l)\right],} \\
& {\left[C_{0}(k), H_{b}\right]=0=\left[C_{0}^{+}(k), H_{b}\right]}
\end{aligned}
$$

for any $k, l \in \mathbb{E}^{3}$, constraints (10) are compatible with the evolution operator equations (7). Moreover, concerning the Hamiltonian operator (4), whose equivalent operator expression is

$H_{b}=\frac{1}{2} \int_{\mathbb{R}^{3}}\left(|E|^{2}+|B|^{2}\right)$,

one has the following result.

\section{4}

The Hamiltonian operator (4) on the Fock subspace $\Phi$ reduced by means of constraints (10) is Hermitian and non-negative definite.

Indeed, by defining the operator

$B(k):=A(k)-\frac{k}{|k|^{2}}\langle k, A(k)\rangle$,

the Hamiltonian operator (4) can be rewritten as

$$
\begin{aligned}
& H_{b}=2 \int_{\mathbb{R}^{3}} d^{3} k|k|^{2}\left\{\left\langle\frac{k}{|k|} \times B^{+}(k), \frac{k}{|k|} \times B(k)\right\rangle+\right. \\
& \left.+\frac{i}{|k|} \varphi(k) C_{0}^{+}(k)+\frac{1}{|k|^{2}}\left[C_{0}^{+}(k)-i|k| \varphi^{+}(k)\right] C_{0}(k)\right\} .
\end{aligned}
$$

The latter, owing to the weak Lorentz-type constraints (10), gives rise to the inequality

$$
\begin{aligned}
& \left(f, H_{b} f\right)=2 \int_{\mathbb{R}^{3}} d^{3} k|k|^{2}\left(\left\langle\frac{k}{|k|} \times B(k) f, \frac{k}{|k|} \times B(k) f\right\rangle\right)= \\
& =2 \int_{\mathbb{R}^{3}} d^{3} k\|k \times B(k) f\|^{2} \geq 0
\end{aligned}
$$

for any vector $f \in \Phi$, proving the proposition.

The Hamiltonian operator expression (4) easily follows $[13,14,31]$ from the well-known relativistic invariant classical Fock-Podolsky electromagnetic Lagrangian

$$
\begin{aligned}
& \mathcal{L}_{b}:=\frac{1}{2} \int_{\mathbb{R}^{3}} d^{3} x\left[\left\langle\nabla \varphi+\frac{1}{c} \frac{\partial A}{\partial \tilde{t}}, \nabla \varphi+\frac{1}{c} \frac{\partial A}{\partial \tilde{t}}\right\rangle-\right. \\
& \left.-\langle\nabla \times A, \nabla \times A\rangle-\left(\frac{1}{c} \frac{\partial \varphi}{\partial \tilde{t}}+\langle\nabla, A\rangle\right)^{2}\right] .
\end{aligned}
$$

Based on the Euler-Lagrange equations corresponding to (16), one finds

$\frac{1}{c^{2}} \frac{\partial^{2} A}{\partial \tilde{t}^{2}}-\Delta A=0, \quad \frac{1}{c^{2}} \frac{\partial^{2} \varphi}{\partial \tilde{t}^{2}}-\Delta \varphi=0$,

whose wave solutions allow one to determine the electromagnetic fields (8) and (9) and to verify that the related Maxwell field equations in vacuum are satisfied, if the Lorentz condition

$C_{0}(\bar{t}, x):=\frac{1}{c} \frac{\partial \varphi}{\partial \tilde{t}}+\langle\nabla, A\rangle=0$

holds for all $(\tilde{t}, x) \in M^{4}$. Moreover, from the Lagrangian expression (16), one easily obtains, by

ISSN 2071-0194. Ukr. J. Phys. 2017. Vol. 62, No. 2 
means of the corresponding Legendre transformation $[2,4,14]$, the Hamiltonian operator

$$
\begin{aligned}
& H_{b}=\frac{1}{2} \int_{\mathbb{R}^{3}} d^{3} x\left(|E|^{2}+|B|^{2}-C_{0}^{2}\right)+ \\
& +\int_{\mathbb{R}^{3}} d^{3} x\left(\langle\nabla, A\rangle^{2}-\langle\nabla \varphi, \nabla \varphi\rangle\right),
\end{aligned}
$$

which is equivalent in the Fock space $\Phi$, modulo solutions (5) of the wave equations (17), to the abovewritten operator expression (4).

Taking the operator equations (7) into account, one easily obtains that

$$
\begin{aligned}
& C_{0}(k)=i[\langle k, A(k)\rangle-|k| \varphi(k)] \neq 0, \\
& C_{0}^{+}(k)=-i\left[\left\langle k, A^{+}(k)\right\rangle-|k| \varphi^{+}(k)\right] \neq 0,
\end{aligned}
$$

contradicting the above-imposed Lorentz constraint (18). Since the latter should vanish in the Fock space, it was suggested in [21] to reduce the Fock space $\Phi$ to a subspace, on which only the weak Lorentz-type operator constraints (10) are satisfied. Concerning these constraints imposed on the Fock space $\Phi$, it is necessary to mention that the corresponding vacuum vector $|0\rangle \in \Phi$ does not, evidently, annihilate the operators $\varphi(k): \Phi \rightarrow \Phi$ and $A^{+}(k): \Phi \rightarrow \Phi^{3}$, as they do not form the pairs commuting with the operators $C_{0}^{+}(k)$ and $C_{0}(k)$, respectively.

\section{The Transformed Fock \\ Space, Its Lorentz-Type Reduction and the Quantum Maxwell Equations}

Since we are interested in describing the selfinteraction of the fermionic quantum particle field $\psi: \Phi \rightarrow \Phi^{4}$ with the field of related self-generated bosonic electromagnetic potentials $(\varphi, A): \Phi \rightarrow \Phi^{4}$, we need, within the Fock multitime approach [21,31], to consider firstly the fermionic particle and bosonic electromagnetic fields in the common reference frame $\mathcal{K}_{t}$ specified by the temporal parameter $t \in \mathbb{R}$. Second, we need to use the classical "minimum interaction" principle [9, 17], (whose sketched backgrounds are presented in Supplement 5) and to apply it to the Hamiltonian operator expression (1):

$H_{f}^{(\mathrm{int})}=\int_{\mathbb{R}^{3}} d^{3} x \psi^{+}\left\langle c \alpha, \frac{\hbar}{i} \nabla\right\rangle \psi+$

ISSN 2071-0194. Ukr. J. Phys. 2017. Vol. 62, No. 2
$+\int_{\mathbb{R}^{3}} d^{3} x\left(\xi \psi^{+} \psi \varphi-\xi \psi^{+}\langle c \alpha, A\rangle \psi\right)$,

in which the fermionic $\psi: \Phi \rightarrow \Phi^{4}$ and bosonic $(\varphi, A): \Phi \rightarrow \Phi^{4}$ operators are commuting a priori to each other as quantum fields of different nature. Since the whole quantum-field system consists of the fermionic particle and bosonic self-generated electromagnetic fields, its evolution is described by means of the joint Hamiltonian operator

$H_{f-b}:=H_{f}^{(\mathrm{int})}+H_{b}$

via the Heisenberg equations

$$
\begin{aligned}
& \frac{\partial \psi}{\partial t}:=\frac{i}{\hbar}\left[H_{f-b}, \psi\right], \frac{\partial \psi^{+}}{\partial t}:=\frac{i}{\hbar}\left[H_{f-b}, \psi^{+}\right], \\
& \frac{\partial A}{\partial t}:=\frac{i}{\hbar}\left[H_{f-b}, A\right], \frac{\partial \varphi}{\partial t}:=\frac{i}{\hbar}\left[H_{f-b}, \varphi\right]
\end{aligned}
$$

with respect to the common temporal parameter $t \in$ $\in \mathbb{R}$. In this case, it is assumed that the corresponding temporal parameters $\tilde{t} \in \mathbb{R}$ and $\bar{t} \in \mathbb{R}$ coincide, that is $\tilde{t}=\bar{t}=t \in \mathbb{R}$ and, by definition, the operator spinor $\psi(t, x):=\left.\psi(\bar{t}, \tilde{t})\right|_{\tilde{t}=\bar{t}=t}$. Simultaneously, the beforederived electromagnetic field evolution equations (7) with respect to the own reference frame $\mathcal{K}_{\bar{t}}$ and the modified fermionic charged particle field equations

$$
\frac{\partial \psi}{\partial \bar{t}}:=\frac{i}{\hbar}\left[H_{f}^{(\mathrm{int})}, \psi\right], \frac{\partial \psi^{+}}{\partial \bar{t}}:=\frac{i}{\hbar}\left[H_{f}^{(\mathrm{int})}, \psi^{+}\right]
$$

with respect to the own reference frame $\mathcal{K}_{\bar{t}}$ should be, evidently, satisfied.

Being mostly interested in the evolution of the quantum particle fermionic field $\psi: \Phi \rightarrow \Phi$, we can get rid of the bosonic Hamiltonian impact into (24), having applied the unitary canonical transformation

$\Phi \rightarrow \tilde{\Phi}:=U(t) \Phi$

to the Fock space $\Phi$. Here, we denoted, by $U(t): \Phi \rightarrow$ $\rightarrow \Phi$, the unitary operator satisfying the determining equation

$d U(t) / d t=\frac{i}{\hbar} H_{b} U(t)$

subject to the bosonic Hamiltonian operator (4) and the temporal parameter $t \in \mathbb{R}$. As a consequence of 
transformation (25), we obtain the effective fermionic particle field interaction Hamiltonian operator

$$
\begin{aligned}
& \tilde{H}_{f}^{(\mathrm{int})}:=U(t) H_{f}^{(\mathrm{int})} U^{*}(t)=\int_{\mathbb{R}^{3}} d^{3} x \psi^{+}\left\langle c \alpha, \frac{\hbar}{i} \nabla\right\rangle \psi+ \\
& +\int_{\mathbb{R}^{3}} d^{3} x\left(\xi \psi^{+} \psi \tilde{\varphi}-\xi \psi^{+}\langle c \alpha, \tilde{A}\rangle \psi\right),
\end{aligned}
$$

where, by definition,

$\left.\tilde{A}:=U(t) A U^{*}(t), \tilde{\varphi}:=U(t) \varphi U^{*}(t)\right]$,

subject to which the evolution in the transformed Fock space $\tilde{\Phi}$, that is induced by the Hamiltonian operator (4)

$$
\begin{aligned}
& \tilde{H}_{b}:=U(t) H_{b} U^{*}(t)= \\
& =2 \int_{\mathbb{R}^{3}} d^{3} k|k|^{2}\left[\left\langle\tilde{A}^{+}(k), \tilde{A}(l)\right\rangle-\tilde{\varphi}(k) \tilde{\varphi}^{+}(k)\right],
\end{aligned}
$$

became completely eliminated. Concerning the Hamiltonian operator (29), we mention that the related commutation relations for the operators $(\tilde{\varphi}(k), \tilde{A}(k)): \tilde{\Phi} \rightarrow \tilde{\Phi}^{4}$ and $\left(\tilde{\varphi}^{+}(k), \tilde{A}^{+}(k)\right): \tilde{\Phi} \rightarrow \tilde{\Phi}^{4}$ remain the same as $(6)$, i.e.,

$$
\begin{aligned}
& {\left[\tilde{\varphi}(k), \tilde{\varphi}^{+}(s)\right]=-\frac{c \hbar}{2|k|} \delta(k-s),\left[\tilde{\varphi}(k), \tilde{A}_{j}(s)\right]=0,} \\
& {\left[\tilde{A}_{j}(k), \tilde{A}_{l}^{+}(s)\right]=\frac{c \hbar}{2|k|} \delta_{j l} \delta(k-s),} \\
& {[\tilde{\varphi}(k), \tilde{\varphi}(s)]=0=\left[\tilde{\varphi}^{+}(k), \tilde{\varphi}^{+}(s)\right],} \\
& {\left[\tilde{A}_{j}(k), \tilde{A}_{l}(s)\right]=0=\left[\tilde{A}_{j}^{+}(k), \tilde{A}_{l}^{+}(s)\right],}
\end{aligned}
$$

for all $k, s \in \mathbb{E}^{3}$ and $j, l \in \overline{1,3}$.

Now, concerning the Hamiltonian operators (27) and (29), the following Heisenberg evolutions equations

$$
\frac{\partial \psi}{\partial \bar{t}}:=\frac{i}{\hbar}\left[\tilde{H}_{f}^{(\mathrm{int})}, \psi\right], \frac{\partial \psi^{+}}{\partial \bar{t}}:=\frac{i}{\hbar}\left[\tilde{H}_{f}^{(\mathrm{int})}, \psi^{+}\right]
$$

with respect to the own reference frame $\mathcal{K}_{\bar{t}}$ and the Heisenberg evolution equations

$\frac{\partial \tilde{\varphi}}{\partial \tilde{t}}:=\frac{i}{\hbar}\left[\tilde{H}_{b} \tilde{\varphi}\right], \quad \frac{\partial \tilde{A}}{\partial \tilde{t}}:=\frac{i}{\hbar}\left[\tilde{H}_{b}, \tilde{A}\right]$

with respect to the own reference frame $\mathcal{K}_{\tilde{t}}$ hold. Being further interested in the evolution equations
(23) suitably rewritten in the transformed Fock space $\tilde{\Phi}$ with respect to the common temporal parameter $t \in \mathbb{R}$, we need to account [31] for that the functional relations

$\psi(t):=\left.\psi(\bar{t}, \tilde{t})\right|_{\bar{t}=\tilde{t}=t}, \tilde{A}(t):=\left.\tilde{A}(\bar{t}, \tilde{t})\right|_{\bar{t}=\tilde{t}=t}$

hold. In particular, relations (33) yield the evolution expressions

$$
\begin{aligned}
& \partial \psi(t) / \partial t=\partial \psi(\bar{t}, \tilde{t}) /\left.\partial \bar{t}\right|_{\bar{t}=\tilde{t}=t}+\partial \psi(\bar{t}, \tilde{t}) /\left.\partial \tilde{t}\right|_{\bar{t}=\tilde{t}=t}, \\
& \partial \tilde{A}(t) / \partial t=\partial \tilde{A}(\bar{t}, \tilde{t}) /\left.\partial \bar{t}\right|_{\bar{t}=\tilde{t}=t}+\partial \tilde{A}(\bar{t}, \tilde{t}) /\left.\partial \tilde{t}\right|_{\bar{t}=\tilde{t}=t}
\end{aligned}
$$

for all $t \in \mathbb{R}$. The latter will be useful, when deriving the resulting quantum Maxwell electromagnetic equations.

Before doing this, we need to consider that the weak operator Lorentz constraints (10) rewritten in the transformed Fock space $\tilde{\Phi}$ are compatible with the evolution equations (32):

$\left[\tilde{C}_{0}(k), \tilde{H}_{b}\right]=0=\left[\tilde{C}_{0}^{+}(k), \tilde{H}_{b}\right]$

However, they fail to be compatible with the evolution equations (31), i.e.,

$\left[\tilde{C}_{0}(k), \tilde{H}_{f}^{(\mathrm{int})}\right] \neq 0 \neq\left[\tilde{C}_{0}^{+}(k), \tilde{H}_{f}^{(\mathrm{int})}\right]$.

This means that we can not impose the constraints

$$
\begin{aligned}
& \tilde{C}_{0}(k) \tilde{\Phi}:=i(\langle k, \tilde{A}(k)\rangle-|k| \tilde{\varphi}(k)) \tilde{\Phi} \neq 0, \\
& \tilde{C}_{0}^{+}(k) \tilde{\Phi}:=-i\left(\left\langle k, \tilde{A}^{+}(k)\right\rangle-|k| \tilde{\varphi}^{+}(k)\right) \tilde{\Phi} \neq 0
\end{aligned}
$$

invariantly for all $k \in \mathbb{E}^{3}$ on the transformed Fock space $\tilde{\Phi}$. Notwithstanding, it is easy enough to verify that the slightly perturbed operators

$$
\begin{aligned}
& \tilde{C}(k):=\tilde{C}_{0}(k)+\frac{i \xi \exp (-i c|k| \bar{t})}{2|k|(2 \pi)^{3 / 2}} \times \\
& \times \int_{\mathbb{R}^{3}} \exp (-i\langle k, y\rangle) \psi^{+}(y) \psi(y) d^{3} y, \\
& \tilde{C}^{+}(k):=\tilde{C}_{0}^{+}(k)-\frac{i \xi \exp (i c|k| \bar{t})}{2|k|(2 \pi)^{3 / 2}} \times \\
& \times \int_{\mathbb{R}^{3}} \exp (i\langle k, y\rangle) \psi^{+}(y) \psi(y) d^{3} y,
\end{aligned}
$$

ISSN 2071-0194. Ukr. J. Phys. 2017. Vol. 62, No. 2 
are mutually commuting with one another and with the Hamiltonian operators (27) and (29):

$$
\begin{aligned}
& {[\tilde{C}(k), \tilde{C}(s)]=0=\left[\tilde{C}^{+}(k), \tilde{C}(s)\right]} \\
& {\left[\tilde{C}(k), \tilde{H}_{f}^{(\mathrm{int})}\right]=0=\left[\tilde{C}^{+}(k), \tilde{H}_{f}^{(\mathrm{int})}\right],} \\
& {\left[\tilde{C}(k), \tilde{H}_{b}\right]=0=\left[\tilde{C}^{+}(k), \tilde{H}_{b}\right]}
\end{aligned}
$$

for all $k, s \in \mathbb{E}^{3}$. Thus, the related evolution flows (31) and $(32)$ in the transformed Fock space $\tilde{\Phi}$ should be considered under the modified constraints

$\tilde{C}(k) \tilde{\Phi}=0=\tilde{C}^{+}(k) \tilde{\Phi}$

for all $k \in \mathbb{E}^{3}$. With regard for the exact expressions (37), constraints (39) can be equivalently rewritten as

$\tilde{C}(\bar{t} ; \tilde{t}, x) \tilde{\Phi}=0$,

where, for all $x \in \mathbb{R}$ and the corresponding temporal parameters $\bar{t}$ and $\tilde{t} \in \mathbb{R}$,

$\tilde{C}(\bar{t} ; \tilde{t}, x):=\frac{1}{(2 \pi)^{3 / 2}} \int_{\mathbb{R}^{3}} d^{3} k \tilde{C}(k) \exp (i\langle k, x\rangle-i|k| \tilde{t})+$ $+\frac{1}{(2 \pi)^{3 / 2}} \int_{\mathbb{R}^{3}} d^{3} k \tilde{C}^{+}(k) \exp (-i\langle k, x\rangle+i|k| \tilde{t})=$

$=\langle\nabla, \tilde{A}\rangle+\frac{1}{c} \frac{\partial \tilde{\varphi}}{\partial \tilde{t}}-\frac{\xi}{2 \pi} \int_{\mathbb{R}^{3}} d^{3} y \Theta(c(\bar{t}-\tilde{t}),|x-y|) \psi^{+}(y) \psi(y)$.

Here, we put, by definition, the relativistic generalized function

$$
\begin{aligned}
& \Theta(c(t-t),|x-y|):= \\
& =\frac{\delta(|x-y|+c(\bar{t}-\tilde{t}))-\delta(|x-y|-c(\bar{t}-\tilde{t}))}{2|x-y|}
\end{aligned}
$$

dual to the well-known generalized solution [13, 75]

$\delta\left(|x-y|^{2}-c^{2}(\bar{t}-\tilde{t})^{2}\right)=$

$=\frac{\delta(|x-y|+c(\bar{t}-\tilde{t}))+\delta(|x-y|-c(\bar{t}-\tilde{t}))}{2|x-y|}$

to the relativistic wave equation.

It is worth to mention that the above-defined operator $\tilde{C}(\bar{t}): \tilde{\Phi} \rightarrow \tilde{\Phi}$ depending parametrically on ISSN 2071-0194. Ukr. J. Phys. 2017. Vol. 62, No. 2 the bosonic temporal parameter $\bar{t} \in \mathbb{R}$ satisfies the relativistic wave equation

$\frac{1}{c^{2}} \frac{\partial^{2} \tilde{C}}{\partial \tilde{t}^{2}}-\Delta \tilde{C}=0$

what can be easily verified, if we use the wave equations (17) rewritten in the Fock space:

$\frac{1}{c^{2}} \frac{\partial^{2} \tilde{A}}{\partial \tilde{t}^{2}}-\Delta \tilde{A}=0, \frac{1}{c^{2}} \frac{\partial^{2} \tilde{\varphi}}{\partial \tilde{t}^{2}}-\Delta \tilde{\varphi}=0$.

Moreover, as can be shown by means of direct calculations, the transformed bosonic Hamiltonian operator (29) on the Fock space $\tilde{\Phi}$ reduced via the modified Lorentz-type constraints (40) persists to be, as before, non-negative definite.

Now, we can proceed to deriving the quantum Maxwell equations, by starting from the operator equations (44) and definitions (8) and (9) of the electromagnetic fields suitably transformed to the Fock space $\tilde{\Phi}$ :

$$
\begin{aligned}
& \left(\nabla \times \tilde{B}-\frac{1}{c} \frac{\partial \tilde{E}}{\partial \tilde{t}}\right) \tilde{\Phi}= \\
& =\frac{\xi}{2 \pi} \nabla \int_{\mathbb{R}^{3}} d^{3} y \Theta(c(\bar{t}-\tilde{t}),|x-y|) \psi^{+}(y) \psi(y) \tilde{\Phi}
\end{aligned}
$$

and

$\langle\nabla, \tilde{E}\rangle \tilde{\Phi}=$

$=-\frac{\xi}{2 \pi} \frac{\partial}{\partial \tilde{t}} \int_{\mathbb{R}^{3}} d^{3} y \Theta(c(\bar{t}-\tilde{t}),|x-y|) \psi^{+}(y) \psi(y) \tilde{\Phi}$,

which are considered in the weak operator sense. In view of relations (32) and (34), we can obtain the following strong operator relations for the electrical and magnetic fields:

$\tilde{E}=-\frac{1}{c} \frac{\partial \tilde{A}}{\partial \tilde{t}}-\nabla \tilde{\varphi}=-\frac{1}{c} \frac{\partial \tilde{A}}{\partial \tilde{t}}-\nabla \tilde{\varphi}, \tilde{B}=\nabla \times \tilde{A}$

with respect to the common reference frame $\mathcal{K}_{t}$. Similarly, one can easily calculate the weak operator relation

$$
\left(\frac{1}{c} \frac{\partial \tilde{\varphi}}{\partial t}+\langle\nabla, \tilde{A}\rangle\right) \tilde{\Phi}=0
$$

which holds for the common temporal parameter $t \in \mathbb{R}$. 
Now, we will calculate the weak Maxwell-type operator relations (45) and (46) with respect to the common reference frame $\mathcal{K}_{t}$ :

$\left.\left(\nabla \times \tilde{B}-\frac{1}{c} \frac{\partial \tilde{E}}{\partial \tilde{t}}\right)\right|_{\bar{t}=\tilde{t}=t} \tilde{\Phi}=\frac{\xi}{2 \pi} \nabla \times$
$\times\left.\int_{\mathbb{R}^{3}} d^{3} y \Theta(c(\bar{t}-\tilde{t}),|x-y|) \psi^{+}(y) \psi(y)\right|_{\bar{t}=\tilde{t}=t} \tilde{\Phi}=0$

and

$\langle\nabla, \tilde{E}\rangle \tilde{\Phi}=-\frac{\xi}{2 \pi} \frac{\partial}{\partial \tilde{t}} \times$

$\times\left.\int_{\mathbb{R}^{3}} d^{3} y \Theta(c(\bar{t}-\tilde{t}),|x-y|) \psi^{+}(y) \psi(y)\right|_{\bar{t}=\tilde{t}=t} \tilde{\Phi}=$

$=\xi \psi^{+} \psi \tilde{\Phi}$,

where we used the known $[14,75]$ generalized functional relation

$\left.\frac{1}{c} \frac{\partial}{\partial s} \Theta(c s,|z|)\right|_{s=0}=-2 \pi \delta(z)$

for all $z \in \mathbb{R}^{3}$. To calculate expression (49), we use the strong operator relations (34) and find that

$\left.\frac{\partial \tilde{E}}{\partial \tilde{t}}\right|_{\bar{t}=\tilde{t}=t}=\frac{\partial \tilde{E}}{\partial t}-\left.\frac{\partial \tilde{E}}{\partial \bar{t}}\right|_{\bar{t}=\tilde{t}=t}=$

$=\frac{\partial \tilde{E}}{\partial t}-\frac{i}{\hbar}\left[\tilde{H}_{f}^{(\mathrm{int})}, \tilde{E}\right]=\frac{\partial \tilde{E}}{\partial t}+\xi \psi^{+} \alpha \psi$.

Thus, relations (52) and (49) yield

$\left(\nabla \times \tilde{B}-\frac{1}{c} \frac{\partial \tilde{E}}{\partial t}\right) \tilde{\Phi}=\xi \psi^{+} \alpha \psi \tilde{\Phi}$

with respect to the common reference frame $\mathcal{K}_{t}$. The combined weak operator relations (50) and (53),

$$
\begin{aligned}
& \left(\nabla \times \tilde{B}-\frac{1}{c} \frac{\partial \tilde{E}}{\partial t}\right) \tilde{\Phi}=\xi \psi^{+} \alpha \psi \tilde{\Phi}, \\
& \langle\nabla, \tilde{E}\rangle \tilde{\Phi}=\xi \psi^{+} \psi \tilde{\Phi}
\end{aligned}
$$

in the Fock space $\tilde{\Phi}$ reduced by the weak constraint (48) jointly with the evident strong operator relations

$\nabla \times \tilde{E}+\frac{1}{c} \frac{\partial \tilde{B}}{\partial \tilde{t}}=0, \nabla \times \tilde{B}=0$

compile the complete system of quantum Maxwell equations with respect to the common reference frame $\mathcal{K}_{t}$.

\section{8}

Really, from the Heisenberg evolution equations (31), one easily obtains the strong operator charge conservative flow relation

$\frac{\partial}{\partial t}\left(\xi \psi^{+} \psi\right)+\left\langle\nabla, \xi \psi^{+} c \alpha \psi\right\rangle=0$,

in which the quantity

$\rho:=\xi \psi^{+} \psi$

is interpreted as the operator charge density, and the quantity

$J:=\xi \psi^{+} c \alpha \psi$

is naturally interpreted as the operator current density in the space $\mathbb{R}^{3}$. From whence, the weak operator equations (54) can be rewritten, in view of definitions (57) and (58), as a weak form of the standard Maxwell equations:

$$
\left.\left(\nabla \times \tilde{B}-\frac{1}{c} \frac{\partial \tilde{E}}{\partial t}\right\rangle\right) \tilde{\Phi}=\frac{J}{c} \tilde{\Phi}, \quad\langle\nabla, \tilde{E}\rangle \tilde{\Phi}=\rho \tilde{\Phi}
$$

under constraint (48) imposed on the Fock space $\tilde{\Phi}$. Moreover, based on the weak operator Maxwell equations (59) and the Lorentz constraint (48), one can derive easily the following weak operator linear wave equations:

$$
\left(\frac{1}{c^{2}} \frac{\partial^{2} \tilde{\varphi}}{\partial t^{2}}-\Delta \tilde{\varphi}\right) \tilde{\Phi}=\rho \tilde{\Phi}, \quad\left(\frac{1}{c^{2}} \frac{\partial^{2} \tilde{A}}{\partial t^{2}}-\Delta \tilde{A}\right) \tilde{\Phi}=\frac{J}{c} \tilde{\Phi}
$$

in respect to the common laboratory reference frame $\mathcal{K}_{t}$, which allows us to calculate the causal quantum bosonic potentials $\left(\tilde{\varphi}_{\xi}, \tilde{A}_{\xi}\right), \tilde{\Phi} \rightarrow \tilde{\Phi}^{4}$, induced by the charged fermionic field in the analytical form:

$\tilde{\varphi}_{\xi}=\frac{1}{4 \pi} \int_{\mathbb{R}^{3}} \frac{\rho\left(t^{\prime}, y\right) d^{3} y}{|x-y|}, \quad \tilde{A}_{\xi}=\frac{1}{4 \pi c} \int_{\mathbb{R}^{3}} \frac{J\left(t^{\prime}, y\right) d^{3} y}{|x-y|}$,

where the "retarded" temporal parameter $t^{\prime}:=t-\mid x-$ $-y \mid / c \in \mathbb{R}$, making Eqs. (60) exactly satisfied modulo by the solutions to their uniform forms. Moreover, owing to (56), expressions (61) satisfy exactly the strong operator Lorentz constraint

$\frac{1}{c} \frac{\partial \tilde{\varphi}_{\xi}}{\partial t}+\left\langle\nabla, \tilde{A}_{\xi}\right\rangle=0$

ISSN 2071-0194. Ukr. J. Phys. 2017. Vol. 62, No. 2 
with respect to the laboratory reference frame $\mathcal{K}_{t}$.

From the analysis of the quantum fermionic field model of a charged particle interacting with the selfgenerated quantum bosonic electromagnetic field, we can infer the following important consequences:

1. The physical effective evolution of the fermionicbosonic system with respect to the common reference frame $\mathcal{K}_{t}$ is governed by the reduced fermionic Hamiltonian operator (27) acting on the canonically transformed Fock space $\tilde{\Phi}$ reduced by means of the weak Lorentz-type operator constraint (48);

2. The compatibility of evolutions of the quantum fermionic and bosonic fields with respect to the common temporal reference frame $\mathcal{K}_{t}$ entails the reciprocal influence of the fermionic field on the bosonic one and vice versa, being clearly demonstrated both by the operator equations (60) for weak field potentials and the Lorentz-type weak constraint (48) imposed on the Fock space $\tilde{\Phi}$;

3. As for the basic self-interacting fermionic-bosonic system described by the joint Hamiltonian operator $(22)$ in the transformed Fock space $\tilde{\Phi}$, we can claim that the bosonic electromagnetic impact into the quantum charged particle dynamics is decisive, since, owing to it, the fermionic system can realize its charge interaction property through the physical vacuum deformation caused by a related deformation of the weak Lorentz-type operator constraint (40) and resulting into the weak operator potential equations (61).

As was shown in [64], the consequences formulated above subject to the quantum fermionic-bosonic selfinteracting phenomenon appear to be very important from the classical point of view, especially for the physical comprehension of the inertial properties of a charged particle under the action of the self-generated electromagnetic field.

\section{Classical Reduction of the Quantum Charged Particle and Electromagnetic Field Evolutions}

Let us consider the vector position operator $\hat{x}: \tilde{\Phi} \rightarrow$ $\rightarrow \tilde{\Phi}^{3}$ and its weak evolution in the reduced Fock space $\tilde{\Phi}$ with respect to the complete suitably renormalized charged particle Hamiltonian operator (27). Taking into account that the Hamiltonian op- erator $\tilde{H}_{f}^{(\mathrm{int})}: \tilde{\Phi} \rightarrow \tilde{\Phi}$ can be represented as

$\tilde{H}_{f}^{(\mathrm{int})}=\int_{\mathbb{R}^{3}} d^{3} x \psi^{+}\left\langle c \alpha, \hat{p}_{x}\right\rangle \psi+$
$+\int_{\mathbb{R}^{3}} d^{3} x\left(\xi \psi^{+} \psi \tilde{\varphi}_{\xi}-\xi \psi^{+}\left\langle c \alpha, \tilde{A}_{\xi}\right\rangle \psi\right)$,

within which the operators $\left(\tilde{\varphi}_{\xi}, \tilde{A}_{\xi}\right): \tilde{\Phi} \rightarrow \tilde{\Phi}^{3}$ are given by the nonlocal integral expressions (61), and $\hat{p}_{x}: \tilde{\Phi} \rightarrow \tilde{\Phi}^{3}$ is the locally defined charged particle $\xi$ momentum operator $\hat{p}_{x}:=\frac{\hbar}{i} \nabla_{x}$ canonically conjugated [7] to the position operator $\hat{x}: \tilde{\Phi} \rightarrow \tilde{\Phi}^{3}$, i.e.,

$\left[\hat{p}_{y}, \hat{x}\right]=\frac{\hbar}{i} \delta(x-y)$

for any $x, y \in \mathbb{R}^{3}$. This means, in particular, that the position operator $\hat{x}: \tilde{\Phi} \rightarrow \tilde{\Phi}^{3}$ is a priori given in the diagonal representation: $\hat{x} \tilde{f}:=x \tilde{f}$ for any vector $\tilde{f} \in \tilde{\Phi}$.

As a result of the simple calculation, we get the expression

$d \hat{x} / d t=\psi^{+} c \alpha \psi$

which can be used to obtain the classical charged particle $\xi$ velocity $u(t, x) \in T\left(\mathbb{R}^{3}\right)$

$u(t, x):=(\Omega, d \hat{x} / d t \Omega)=\left(\Omega, \psi^{+} c \alpha \psi \Omega\right)$,

where the vector $\Omega \in \tilde{\Phi}$ is the ground state of the Hamiltonian operator (63) acting in the Lorentz-type Fock space $\tilde{\Phi}$ reduced and suitably renormalized $[7$, 13, 14,70]. Substituting (65) and (57) into the Hamiltonian expression (63), we obtain the expression

$$
\begin{aligned}
& \tilde{H}_{f}^{(\mathrm{int})}=\int_{\mathbb{R}^{3}} d^{3} x\left\langle d \hat{x} / d t, \hat{p}_{x}\right\rangle+ \\
& +\int_{\mathbb{R}^{3}} d^{3} x\left(\rho \tilde{\varphi}_{\xi}-\left\langle\frac{1}{c} J, \tilde{A}_{\xi}\right\rangle\right),
\end{aligned}
$$

whose classical counterpart looks as

$$
\bar{H}_{f}^{(\mathrm{int})}=\int_{\mathbb{R}^{3}} d^{3} x\left(\rho \tilde{\varphi}_{\xi}-\left\langle\frac{1}{c} J, \tilde{A}_{\xi}\right\rangle\right),
$$

within which we took the previously assumed quantum massless charged particle $\xi$ fermionic field into 
account. Expression (68) jointly with the renormalized bosonic field Hamiltonian (12) gives rise to the complete classical Hamiltonian function

$\bar{H}_{f-b}^{(\mathrm{int})}=\int_{\mathbb{R}^{3}} d^{3} x\left[\frac{1}{2}\left(|\tilde{E}|^{2}+|\tilde{B}|^{2}\right)+\rho \tilde{\varphi}_{\xi}-\left\langle\frac{1}{c} J, \tilde{A}_{\xi}\right\rangle\right]$

governing the temporal evolution of the charged particle $\xi$ and the electromagnetic field with respect to the laboratory reference frame $\mathcal{K}_{t}$. The above-obtained Hamiltonian function and its corresponding Lagrangian form (16) have been effectively used before in [64] to describe the classical selfinteracting charged particle dynamics and its inertial properties.

Being experienced with the analysis of a selfinteracting charged quantum particle fermionic field with the self-generated quantum bosonic electromagnetic field, we understand well that the influence of the electromagnetic field on the charged particle should be considered as crucial, strongly modifying the related fermionic Hamiltonian operator describing the charged particle dynamics. Since the simultaneously modified bosonic electromagnetic operator depends, owing to the self-interaction, on the charge and current particle field densities, the joint impact on the charged particle dynamics can be efficiently classically modeled by means of its inertial mass parameter. In the quantum operator case, the physical charged particle mass parameter $m_{\mathrm{ph}} \in$ $\mathbb{R}_{+}$can be naturally defined by means of the least eigenvalue of the quantum renormalized Hamiltonian (22),

$$
\begin{aligned}
& m_{\mathrm{ph}}:=c^{-2} \inf _{\tilde{f} \in \tilde{\Phi},\|\tilde{f}\|=1}\left(\tilde{f}, \tilde{H}_{f-b}^{(\mathrm{int})} \tilde{f}\right), \\
& \tilde{H}_{f-b}^{(\mathrm{int})}:=\tilde{H}_{f}^{(\mathrm{int})}+\tilde{H}_{b},
\end{aligned}
$$

acting in the Fock space $\tilde{\Phi}$ suitably transformed and reduced by means of the operator Lorentz-type constraint (48) with respect to the common reference frame $\mathcal{K}_{t}$. The very complicated quantum spectral problem (70) was recently analyzed in [64] from the classical point of view in detail. A thorough analytical study of the mass expression (70) is under elaboration.

\section{Conclusion}

Within the framework of the Fock multi-time parameter approach and the Feynman proper-time paradigm, a quantum relativistic fermionic massless charged particle self-interacting with the self-generated bosonic electromagnetic field was reanalyzed. The self-interaction Fock space phenomenon structure was discussed in detail. We have shown that the quantum mechanism of self-interaction of a charged particle with its self-generated electromagnetic field consists of two physically different phenomena. Their influence on the structure of the resulting Hamilton interaction operator appeared to be crucial for determining the related charged particle mass spectrum.

The author conveys his cordial thanks to Prof. Hal Puthoff (Institute for Advanced Studies at Austin, Texas, USA) for sending his original works, and to Prof. Roman Jackiw (Department of Physics at the Massachusetts Institute of Technology, MT, USA) for instrumental discussions during the collaborative research stay at the NJIT, NJ, USA, May 25-31, 2015, as well as for the related comments and useful remarks. He is also very indebted to Prof. Denis Blackmore (NJIT, Newark, NJ, USA) and Prof. Edward Kapuscik (Institute for Nuclear Physics at PAS, Kraków, Poland) for the friendly cooperation and important discussions. Especially, he is grateful to the AGH of Krakow (Poland) for partially supporting this research and a travel fund for the SIAM Conference on "Dynamical Systems" held in May 20-25, 2015. The last, but not least thanks belong to the anonymous Referee, whose remarks and suggestions were so helpful for improving the exposition of the article.

\section{SUPPLEMENT:}

"Minimum" Interaction Principle and Its Geometric Backgrounds

In this Supplement, we will sketch the analytical backgrounds of the "minimum" interaction principle widely used in modern theoretical and mathematical physics. For the description of a moving point charged particle under an external electromagnetic field, we will use the geometric approach [2]. Namely, let a trivial fiber bundle structure $\pi: \mathcal{M} \rightarrow \mathbb{R}^{3}, \mathcal{M}=\mathbb{R}^{3} \times G$, with the Abelian structure group $G:=\mathbb{R} \backslash\{0\}$ equivariantly act on the canonically symplectic coadjoint space $T^{*}(\mathcal{M})$. The latter possesses the canonical symplectic structure

$$
\begin{aligned}
& \omega^{(2)}(p, z ; x, g):=d\left(p r_{*}\right)^{*} \alpha^{(1)}(x, g)=\langle d p, \wedge d x\rangle+ \\
& +\left\langle d z, \wedge g^{-1} d g\right\rangle_{\mathcal{G}}+\left\langle z d g^{-1}, \wedge d g\right\rangle_{\mathcal{G}}
\end{aligned}
$$


for all $(p, z ; x, g) \in T^{*}(\mathcal{M})$, where $\alpha^{(1)}(x, g):=\langle p, d x\rangle+$ $+\left\langle z, g^{-1} d g\right\rangle_{\mathcal{G}} \in T^{*}(\mathcal{M})$ is the corresponding Liouville form on $T^{*}(\mathcal{M})$, and $\langle\cdot, \cdot\rangle$ is the usual scalar product in $\mathbb{E}^{3}$. On the fibered space $\mathcal{M}$, one can define a connection $\Gamma$ by means of a one-form $\mathcal{A}: M \rightarrow T^{*}(\mathcal{M}) \times \mathcal{G}$ determined as

$\mathcal{A}(x, g):=g^{-1}\langle\xi A(x), d x\rangle g+g^{-1} d g$

with $\xi \in \mathcal{G}^{*},(x, g) \in \mathbb{R}^{3} \times G$. The corresponding curvature 2-form $\Sigma^{(2)} \in \Lambda^{2}\left(\mathbb{R}^{3}\right) \otimes \mathcal{G}$ is

$\Sigma^{(2)}(x):=d \mathcal{A}(x, g)+\mathcal{A}(x, g) \wedge \mathcal{A}(x, g)=$

$=\xi \sum_{i, j=1}^{3} F_{i j}(x) d x^{i} \wedge d x^{j}$,

where

$F_{i j}(x):=\frac{\partial A_{j}}{\partial x^{i}}-\frac{\partial A_{i}}{\partial x^{j}}$

for $i, j=\overline{1,3}$ is the spatial electromagnetic tensor with respect to the reference frame $\mathcal{K}_{t}$. For an element $\xi \in \mathcal{G}^{*}$ to be compatibly fixed, we need to construct the related momentum mapping $l: T^{*}(\mathcal{M}) \rightarrow \mathcal{G}^{*}$ with respect to the canonical symplectic structure $(71)$ on $T^{*}(\mathcal{M})$ and put, by definition, $l(x, p):=\xi \in \mathcal{G}^{*}$ to be constant, $P_{\xi}:=l^{-1}(\xi) \subset T^{*}(\mathcal{M})$ and $G_{\xi}=\left\{g \in G: A d_{G}^{*} \xi\right\}$ to be the corresponding isotropy group of the element $\xi \in \mathcal{G}^{*}$. Next, we can apply the standard [2,4,9] invariant Marsden-Weinstein-Meyer reduction scheme to the orbit factor space $\tilde{P}_{\xi}:=P_{\xi} / G_{\xi}$ subject to the corresponding group $G$ action. Then, as a result of the Marsden-WeinsteinMeyer reduction, we get that, as $G_{\xi} \simeq G$, the factor-space $\tilde{P}_{\xi} \simeq T^{*}\left(\mathbb{R}^{3}\right)$ becomes a Poisson space with the suitably reduced symplectic structure $\bar{\omega}_{\xi}^{(2)} \in T^{*}\left(\tilde{P}_{\xi}\right)$. The corresponding Poisson brackets on the reduced manifold $\tilde{P}_{\xi}$ equal

$$
\begin{aligned}
& \left\{x^{i}, x^{j}\right\}_{\xi}=0, \quad\left\{p_{j}, x^{i}\right\}_{\xi}=\delta_{j}^{i}, \\
& \left\{p_{i}, p_{j}\right\}_{\xi}=\xi F_{i j}(x)
\end{aligned}
$$

for $i, j=\overline{1,3}$, being considered with respect to the laboratory reference frame $\mathcal{K}_{t}$. Based on (75), it is worth to observe that a new so-called "shifted" momentum variable

$\tilde{\pi}:=p+\xi A(x)$

on $\tilde{P}_{\xi}$ gives rise to the symplectomorphic transformation $\bar{\omega}_{\xi}^{(2)} \rightarrow \tilde{\omega}_{\xi}^{(2)}:=\langle d \tilde{\pi}, \wedge d x\rangle \in \Lambda^{2}\left(T^{*}\left(\mathbb{R}^{3}\right)\right)$. The latter gives rise to the following "minimal interaction" canonical Poisson brackets important in theoretical physics:

$$
\left\{x^{i}, x^{j}\right\}_{\tilde{\omega}_{\xi}^{(2)}}=0,\left\{\tilde{\pi}_{j}, x^{i}\right\}_{\tilde{\omega}_{\xi}^{(2)}}=\delta_{j}^{i},\left\{\tilde{\pi}_{i}, \tilde{\pi}_{j}\right\}_{\tilde{\omega}_{\xi}^{(2)}}=0
$$

for $i, j=\overline{1,3}$, represented already with respect to some new reference frame $\tilde{\mathcal{K}}_{t^{\prime}}$ characterized by the phase space coordinates $(x, \tilde{\pi}) \in \tilde{P}_{\xi}$ and a new evolution parameter $t^{\prime} \in \mathbb{R}$, since the spatial Maxwell field compatibility equations

$\partial F_{i j} / \partial x_{k}+\partial F_{j k} / \partial x_{i}+\partial F_{k i} / \partial x_{j}=0$

are identically satisfied on $\mathbb{R}^{3}$ for all $i, j, k=\overline{1,3}$, owing to the definition of the electromagnetic curvature tensor (74).
1. M. Abraham. Dynamik des electrons in Nachrichten von der Geselschaft der Wissenschafften zu Göttingen (Mathematisch-Physikalische Klasse, 1902), s. 20

2. R. Abraham, J. Marsden. Foundations of Mechanics (Benjamin Cummings, 1987).

3. A. Annila. The meaning of mass. Intern. J. Theor. Math. Phys. 2 (4), 67 (2012) [DOI: 10.5923/j.ijtmp.20120204.03].

4. V.I. Arnold. Mathematical Methods of Classical Mechanics (Springer, 1978).

5. B. Di Bartolo, Classical Theory of Electromagnetism (World Scientific, 2004).

6. R. Becker. Theorie der Elektrizitat. Bd. II. Elektronentheorie (Teubner, 1933)

7. J.D. Bjorken, S.D. Drell. Relativistic Quantum Fields (McGraw-Hill, 1965).

8. D. Blackmore, N.N. Bogolubov (jr.), A.K. Prykarpatski. The Lagrangian and Hamiltonian aspects of the electrodynamic vacuum-field theory models. Boson Journal of Modern Physics (BJMP) 2 (2), 105 (2016).

9. D. Blackmore, A.K. Prykarpatski, V.Hr. Samoylenko. Nonlinear Dynamical Systems of Mathematical Physics: Spectral and Differential-Geometrical Integrability Analysis (World Scientific, 2011).

10. D. Blackmore, A.K. Prykarpatski, N.N. Bogolubov (jr.), J.J. Sławianowski. Mathematical foundations of the classical Maxwell-Lorentz electrodynamic models in the canonical Lagrangian and Hamiltonian formalisms. Universal J. Phys. Appl. 1 (2), 160 (2013) [DOI: 10.13189/ ujpa.2013.010216].

11. K. Bleuer. Eine neue Methode zum Behandlung der longitudinalen und skalaren Photonen. Helv. Phys. Acta 23, 567 (1950).

12. N.N. Bogolubov, N.N. Bogolubov (jr.). Introduction into Quantum Statistical Mechanics (World Scientific, 1986) [ISBN-13: 978-9814295826, ISBN-10: 9814295825].

13. N.N. Bogolubov, A.A. Logunov, A.I. Oksak, I.T. Todorov. General Principles of Quantum Field Theory (Kluwer, 1990) [ISBN: 978-94-010-6707-2].

14. N.N. Bogoliubov, D.V. Shirkov. Quantum Fields (AddisonWesley, 1982).

15. A.K. Prykarpatsky, N.N. Bogolubov (jr.). The Maxwell electromagnetic equations and the Lorentz type force derivation - The Feynman approach legacy. Int. J. Theor. Phys. 5, 237 (2012) [DOI: 10.1007/s10773-011-0900-1].

16. N.N. Bogolubov (jr.), A.K. Prykarpatsky. The analysis of Lagrangian and Hamiltonian properties of the classical relativistic electrodynamics models and their quantization. Found. Phys. 40, 469 (2010) [DOI: 10.1007/s10701-0099399-1].

17. N.N. Bogolubov (jr.), A.K. Prykarpatsky, D. Blackmore. Maxwell-Lorentz electrodynamics revisited via the Lagrangian formalism and Feynman proper time paradigm. Mathematics 3, 190 (2015) [DOI: 10.3390/math3020190].

18. N.N. Bogolubov (jr.), A.K. Prykarpatsky, U. Taneri, Y.A. Prykarpatsky. The electromagnetic Lorentz condition problem and symplectic properties of Maxwell- and 
Yang-Mills-type dynamical systems. J. Phys. A: Math. Theor. 42, 165401 (2009) [DOI: 10.1088/1751-8113/42/16/ 165401].

19. L. Brillouin. Relativity Reexamined (Academic Press, 1970).

20. P.A.M. Dirac. An extensible model of the electron. Proc. Roy. Soc. Ser. A 167, 148 (1938); Proc. R. Soc. Lond. Ser. A 167, 148 (1938).

21. P.A.M. Dirac, V.A. Fock, B. Podolsky. On quantum electrodynamics. Sow. Phys. 2, 468 (1932).

22. F.J. Dyson. Feynman's proof of the Maxwell equations. Am. J. Phys. 58, 209 (1990).

23. F.J. Dyson. Feynman at Cornell. Phys. Today 42 (2), 32 (1989) [DOI: 10.1063/1.881190].

24. A. Einstein. Relativitätsprinzip und die aus demselben gezogenen Folgerungen. Jahrbuch Radioaktivität 4, 411 (1907).

25. E. Fermi. Quantum theory of radiation. Rev. Mod. Phys. 4, 87 (1932) [DOI: 10.1103/RevModPhys.4.87].

26. H.G. Dehmlet. Nobel Lecture. Experiments with an Isolated Subatomic Particle at Rest (World Scientific, 1993).

27. R. Feynman. Statistical Mechanics (Perseus Books, 1998) [ISBN-13: 978-0201360769, ISBN-10: 0201360764].

28. R. Feynman, R. Leighton, M. Sands. The Feynman Lectures on Physics. Electrodynamics (Addison-Wesley, 1964), Vol. 2.

29. R. Feynman, R. Leighton, M. Sands. The Feynman Lectures on Physics. The Modern Science on the Nature. Mechanics. Space, Time, Motion (Addison-Wesley, 1963), Vol. 1.

30. V. Fock. Die eigenzeit in der klassischen und in der quantenmechanik. Sow. Phys. 12, 404 (1937).

31. V.A. Fock, B. Podolsky. On the quantization of electromagnetic waves and the interaction of charges in Dirac's theory. Sow. Phys. 1, 801 (1932).

32. T.L. Gill, W.W. Zachary. Two mathematically equivalent versions of Maxwell equations, Preprint (University of Maryland, 2008)

33. T.L. Gill, W.W. Zachary. Two mathematically equivalent versions of Maxwell's equations. Found. of Physics 4, 99 (2011).

34. T.L. Gill, W.W. Zachary, J. Lindsey. The classical electron problem. Found. of Physics 31 (9), 1299 (2001) [DOI: 10.1023/A:1012222227710].

35. C. Godbillon. Geometrie Differentielle et Mecanique Analytique (Hermann, 1969).

36. S.N. Gupta. Theory of longitudinal photons in quantum electrodynamics. Proc. Phys. Soc. (London) A63, 681 (1950).

37. R.T. Hammond. Electrodynamics and radiation reaction. Found. of Physics 43, 201 (2013) [DOI: 10.1007/s10701012-9687-z].

38. R.T. Hammond. Relativistic particle motion and radiation reaction. Electr. J. Theor. Phys. No. 23, 221 (2010).

39. P. Higgs. Broken symmetries and the masses of gauge bosons. Phys. Rev. Lett. 13, 508 (1964) [DOI: 10.1103/
PhysRevLett.13.508]; Spontaneous symmetry breakdown without massless bosons. Phys. Rev. 145, 1156 (1966) [DOI: 10.1103/PhysRev.145.1156].

40. G. t'Hooft. Renormalizable Lagrangians for massive YangMills fields. Nucl. Phys. B 35, 167 (1971) [DOI: 10.1016/0550-3213(71)90139-8].

41. K. Huang. Statistical Mechanics (Wiley, 1987) [ISBN: 0471-81518-7].

42. J.D. Jackson. Classical Electrodynamics (Wiley, 1999) [ISBN: 978-0-471-30932-1].

43. M. Jammer. Concepts of Mass in Contemporary Physics and Philosophy (Princeton Univ. Press, 2009) [ISBN: 9781400823789].

44. B.P. Kosyakov. Radiation in electrodynamics and in YangMills theory. Sov. Phys. Usp. 35 (2), 135 (1992) [DOI: 10.1070/PU1992v035n02ABEH002218].

45. B.P. Kosyakov. Introduction to the Classical Theory of Particles and Fields (Springer, 2007) [ISBN 978-3-54040934-2]

46. B.A. Kupershmidt, Infinite-dimensional analogs of the minimal coupling principle and of the Poincaré lemma for differential two-forms. Diff. Geom. É Appl. 2, 275 (1992) [DOI: 0.1016/0926-2245(92)90015-F].

47. L.D. Landau, E.M. Lifshitz. The Classical Theory of Fields (Pergamon, 1983).

48. H.A. Lorentz. Electromagnetic phenomena in a system moving with any velocity smaller than that of light. Proceed. of the Royal Netherlands Academy of Arts and Sciences 6, 809 (1904).

49. H.A. Lorentz. Theory of Electrons (Teubner, 1909).

50. H.A. Lorentz. The Theory of Electrons and Its Applications to the Phenomena of Light and Radiant Heat (Jacques Gabay, 1992).

51. A.A. Martins, M.J. Pinheiro. On the electromagnetic origin of inertia and inertial mass. Int. J. Theor. Phys. 47, 2706 (2008) [DOI: 10.1007/s10773-008-9709-y].

52. R. Medina. Radiation reaction of a classical quasi-rigid extended particle. J. Phys. A: Math. Gen. 39, 3801 (2006) [DOI: 10.1088/0305-4470/39/14/021].

53. H. Minkowski. Raum und Zeit. Physik. Z. 10, 104 (1909)

54. V.B. Morozov. On the question of the electromagnetic momentum of a charged body. Physics-Uspekhi 54 (4), 371 (2011) [DOI: 10.3367/UFNr.0181.201104c.0389].

55. A. Ori, E.J. Rosenthal. Calculation of the self force using the extended-object approach. Math. Phys. 45, 2347 (2004) [DOI: $10.1063 / 1.1737052]$.

56. A. Ori, E.J. Rosenthal. Universal self-force from an extended object approach. Phys. Rev. D 68, 041701(R) (2003) [DOI: 10.1103/PhysRevD.68.041701].

57. L. Page, N.I. Adams (jr.). Action and reaction between moving charges. Am. J. Phys. 13, 141 (1945) [DOI: 10.1119/1.1990689].

58. P.T. Pappas. The original Ampère force and Biot-Savart and Lorentz force. Nuovo Cim. B 76 (2), 189 (1983) [DOI: 10.1007/BF02721552].

59. W. Pauli. Theory of Relativity (Oxford Univ. Press, 1958) [ISBN: 978-0486641522].

ISSN 2071-0194. Ukr. J. Phys. 2017. Vol. 62, No. 2 
60. D.T. Pegg. Absorber theory of radiation. Rep. Prog. Phys. 38, 1339 (1975) [DOI: 10.1088/0034-4885/38/12/001].

61. H. Poincaré. Sur la dynamique de l'électron. C.R. Acad. Sci. (Paris) 140, 1504 (1905).

62. A.K. Prykarpatsky. Classical electromagnetic theory revisiting: The A.M. Ampére law and the vacuum field theory approach. Universal J. Phys. Appl. 2 (8), 381 (2014) [DOI: 10.13189/ujpa.2014.020804].

63. A.K. Prykarpatsky, N.N. Bogolubov (jr.), U. Taneri. The relativistic electrodynamics least action principles revisited: New charged point particle and hadronic string models analysis. Int. J. Theor. Phys. 49, 798 (2010) [DOI: 10.1007/s10773-010-0260-2].

64. A.K. Prykarpatsky, N.N. Bogolubov (jr.). On the classical Maxwell-Lorentz electrodynamics, the electron inertia problem, and the Feynman proper time paradigm. Ukr. J. Phys. 61, 187 (2016) [DOI: 10.15407/ujpe61.03.0187].

65. H.E. Puthoff. Casimir vacuum energy and the semicalssical electron. Int. J. Theor. Phys. 46, 3005 (2007) [DOI: 10.1007/s10773-007-9414-2].

66. F. Rohrlich. Classical Charged Particles (Addison-Wesley, 1965) [ISBN: 0201064928].

67. F. Rohrlich. Self-energy and stability of the classical electron. Amer. J. of Phys., 28, 639 (1960).

68. G. Rousseaux. The gauge non-invariance of classical electromagnetism. arXiv:physics/0506203v1 (2005).

69. G. Rousseaux. On the physical meaning of the gauge conditions of classical electromagnetism: The hydrodynamics analogue viewpoint. arXiv:physics/0511047v1 [physics.class-ph] (2005).

70. J. Schwinger. Quantum Electrodynamics (Dover, 1958) [ISBN: 0486604446].

71. J. Schwinger. On gauge invariance of vacuum polarization. Phys. Rev. 82 (5), 664 (1951) [DOI: 10.1103/PhysRev.82.664].
72. J.J. Sławianowski. Geometry of Phase Spaces (Wiley, 1991).

73. V.M. Simulik. The electron as a system of classical electromagnetic and scalar fields. In: What is the Electron? Edited by V.M. Simulik (Apeiron, 2005), p. 109.

74. C. Teitelboim. Splitting of the Maxwell tensor: Radiation reaction without advanced fields. Phys. Rev. D1, 1572 (1970) [DOI: 10.1103/PhysRevD.1.1572, 10.1103/PhysRevD.2.1763]

75. V.S. Vladimirov. Equations of Mathematical Physics (Dekker, 1971) [ISBN: 0824717139].

76. J.B. Wheeler, R.P. Feynman. Interaction with the absorber as the mechanism of radiation. Rev. Modern Phys. 17, 157 (1945) [DOI: 10.1103/RevModPhys.17.157]

77. F. Wilczek. Origins of mass. Open Phys. 10 (5), 1021 (2012) [DOI: 10.2478/s11534-012-0121-0]

78. F. Wilczek. QCD and natural philosophy. Ann. Henry Poincaré 4, 211 (2003) [DOI: 10.1007/s00023-003-0917-y].

79. Y. Yaremko, V. Tretyak. Radiation Reaction in Classical Field Theory (LAMBERT Acad. Publ., 2012).

Received 31.05.16

А.К. Прикарпатсъкий

ПРОБЛЕМА САМОДІЇ ЗАРЯДЖЕНОЇ

ФЕРМІОННОЇ ЧАСТИНКИ В РАМКАХ

БАГАТОЧАСОВОЇ ПАРАДИГМИ ФОКА

ТА ПАРАДИГМИ ВЛАСНОГО ЧАСУ ФЕЙНМАНА

$\mathrm{P}$ е $з$ ю м е

Аналізується самодія квантової безмасової зарядженої ферміонної частинки через власне генероване бозонне електромагнітне поле в рамках багаточасової парадигми Фока та парадигми власного часу Фейнмана. Структура явища самодії вивчається на основі методу, що базується на відповідно ренормалізованому квантовому просторі Фока. Дискутується також спектр мас зарядженої частинки. 